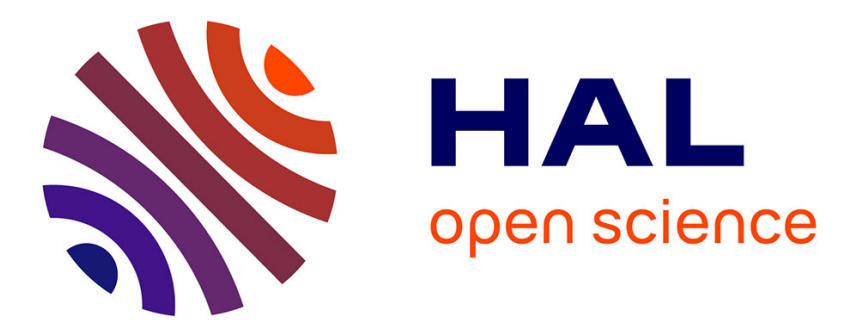

\title{
Analytical iron loss model for the optimization of magnetic gear
}

Melaine Desvaux, Bernard Multon, Stéphane Sire, Hamid Ben Ahmed

\section{To cite this version:}

Melaine Desvaux, Bernard Multon, Stéphane Sire, Hamid Ben Ahmed. Analytical iron loss model for the optimization of magnetic gear. IEEE International Electric Machines and Drives Conference (IEMDC), 2017, May 2017, Miami, United States. pp.1 - 8, 10.1109/IEMDC.2017.8002255 . hal01598123

\section{HAL Id: hal-01598123 \\ https://hal.science/hal-01598123}

Submitted on 29 Sep 2017

HAL is a multi-disciplinary open access archive for the deposit and dissemination of scientific research documents, whether they are published or not. The documents may come from teaching and research institutions in France or abroad, or from public or private research centers.
L'archive ouverte pluridisciplinaire HAL, est destinée au dépôt et à la diffusion de documents scientifiques de niveau recherche, publiés ou non, émanant des établissements d'enseignement et de recherche français ou étrangers, des laboratoires publics ou privés. 


\title{
Analytical Iron Loss Model for the Optimization of Magnetic Gear
}

\author{
Melaine Desvaux ${ }^{1}$, Bernard Multon ${ }^{1}$, Stéphane Sire ${ }^{2}$, Hamid Ben Ahmed ${ }^{1}$ \\ ${ }^{1}$ SATIE, ENS Rennes, Université Bretagne Loire, CNRS, 35170 Bruz, France, melaine.desvaux @ens-rennes.fr \\ ${ }^{2}$ IRDL, Université de Bretagne Occidentale, FRE CNRS 3744, 29200 Brest, France, stephane.sire@ univ-brest.fr
}

\begin{abstract}
This article deals with analytical models dedicated to iron losses in yokes and pole pieces of a magnetic gear with a concentric structure. The magnetic field distribution is determined in yokes by solving both Poisson's and Laplace's equations, whereas for pole pieces the magnetic field is computed by coupling the previous analytical model with a reluctance network model. The iron loss can then be determined in postprocessing from the magnetic field analytical computation. The iron loss model used in this article takes into account the temporal and spatial variations of flux density. Results of this global analytical model permit to analyze the temporal and spatial evolution of flux density. In recognizing that the purpose of this model is to be integrated into a set of models for the global mechatronic optimization of magnetic gears, it is essential to minimize computation time. This work is being applied in the context of high-power (multi-MW) wind turbines.
\end{abstract}

Keywords - Analytical model; Computation time; Flux density; Iron losses; Locus; Magnetic gear; Permeance network; Pole pieces; Yokes.

\section{NOMENCLATURE}

$A^{(k)}$ : Magnetic vector potential of region $\mathrm{k}[\mathrm{Wb}]$

$B_{r}^{(k)}$ : Radial flux density in region $\mathrm{k}[\mathrm{T}]$

$B_{\alpha}^{(k)}$ : Tangential flux density in region $\mathrm{k}[\mathrm{T}]$

$B_{/ /}$: Major axis of the flux density locus [T]

$B_{\perp}$ : Minor axis of the flux density locus [T]

$F_{\text {int }}$ : Iron loss frequency in the internal yoke $[\mathrm{Hz}]$

$F_{\text {ext }}$ : Iron loss frequency in the external yoke $[\mathrm{Hz}]$

$F_{Q}$ : Iron loss frequency in pole pieces $[\mathrm{Hz}]$

$G_{m}$ : Gear ratio

$k$ : Index of the magnetic gear region

$k_{h}$ : Hysteresis coefficient

$k_{e}$ : Eddy current coefficient

$k_{e x}:$ Excess loss coefficient

$L$ : Length of the magnetic gear [m]

$M^{(k)}$ : Radial magnetization distribution of region $\mathrm{k}[\mathrm{Wb}]$

$P_{\text {iron }}$ : Instantaneous iron loss density $\left[\mathrm{W} / \mathrm{m}^{3}\right]$

$p_{\text {int }}:$ Number of internal ring pole pairs

$p_{\text {ext }}$ : Number of external ring pole pairs

$P_{r}:$ Radial permeance

$P_{\alpha}$ : Tangential permeance

$Q:$ Number of ferromagnetic pole pieces

$r$ : Radial cylindrical coordinate [m]

$R_{M}^{(k)}$ : External radius of region $\mathrm{k}[\mathrm{m}]$

$R_{m}^{(k)}$ : Internal radius of region $\mathrm{k}[\mathrm{m}]$
$T$ : Iron loss periodicity [s]

$U_{i, j}$ : Potential of the permeance network for point $i, j$

$Z$ : Matrix system of the analytical model derived from the resolution of Poisson's and Laplace's equations

$\alpha$ : Angular cylindrical coordinate [rad]

$\beta$ : Opening angle of the pole pieces $[\mathrm{rad}]$

$\gamma$ : Steinmetz coefficient

$\xi_{i, j}$ : Major axis angle of the flux density at point $i, j[\mathrm{rad}]$

$\theta:$ Global angular position of the magnetic gear [rad]

$\theta_{i, j}^{*}$ : Global angular position maximizing the flux density at point $i, j[\mathrm{rad}]$

$\mu_{(k)}$ : Relative permeability of region $\mathrm{k}$

$\phi_{r, i, j}$ : Radial flux source of point $i, j$

$\phi_{\alpha, i, j}$ : Tangential flux source of point $i, j$

$\Omega_{\text {int } / 0}$ : Rotational speed of the internal ring [rad/s]

$\Omega_{\text {ext } / 0}$ : Rotational speed of the external ring $[\mathrm{rad} / \mathrm{s}]$

$\Omega_{Q / 0}$ : Rotational speed of the pole piece ring [rad/s]

\section{INTRODUCTION}

Mechanical gearboxes, as currently used in an indirect drive, electromechanical conversion chain (see Fig. 1a), result in a smaller capital expenditure and lower mass than the direct drive conversion chain [1] (Fig. 1b). On the downside, mechanical gearboxes cause production interruptions and require repairs, thus increasing operating costs [2],[3]. In this context, one attractive solution consists of developing a conversion chain featuring a medium-speed generator and a magnetic gear [4] (with non-contact power transmission) (Fig. 1c). The most popular magnetic gear topology was proposed by Martin [5] and has been the topic of various studies conducted by Atallah [6],[7].
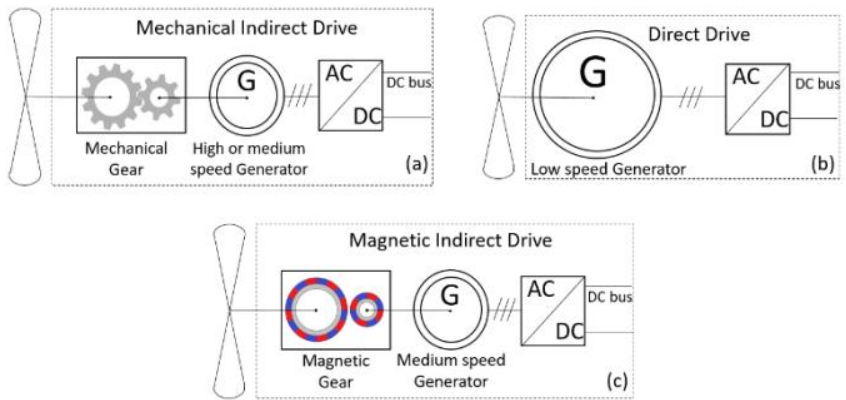

Fig. 1. Conversion chain: a) Indirect mechanical drive, b) Direct drive, c) Indirect magnetic drive (i.e. the one studied here). 
The magnetic gear [7] shown in Fig. 2 potentially offers high performance with a higher torque density and greater reliability than mechanical gearboxes [8]. This gear becomes even more attractive for high-torque applications, like a high-power wind turbine (on the order of several MN.m and several MW) [9]. The competitiveness of this magnetic gear must be evaluated in the context of wind turbine operations, where conversion chain efficiency and heat dissipation constitute major criteria [10]. It then becomes necessary to compute iron losses in both yokes and pole pieces. These losses have already been computed from a magnetic field computation with the finite element method [11],[12]. However, the computation time is too long to evaluate iron losses in a high-power magnetic gear for the purpose of integrating the iron loss computation into a set of models for the global mechatronic optimization of magnetic gears. Moreover, to the best of our knowledge, the iron loss computation method used in other works, e.g. [11],[12], does not take into account the spatial variations of flux density despite the magnitude of such variations.

The major contribution of this article concerns the development of an analytical model that enables computing the iron loss. The iron loss model takes into account both the spatial and temporal variations of the flux density in pole pieces and yokes of the magnetic gears while computing the iron loss more quickly than the finite element method [11],[12]. For yokes, the analytical magnetic field computation is based on the solution to Laplace's and Poisson's equations [13],[14]. For the pole pieces, a previous analytical model is coupled with a bi-directional permeance network model [15] in order to determine the magnetic field distribution. The temporal and spatial evolution of the flux density is evaluate in post-processing to compute iron losses, in conjunction with the conventional electrical machine, in ferromagnetic parts [16], as opposed to [11],[12], which only take temporal variations into consideration.

\section{MAGNETIC GEAR PRINCIPLE}

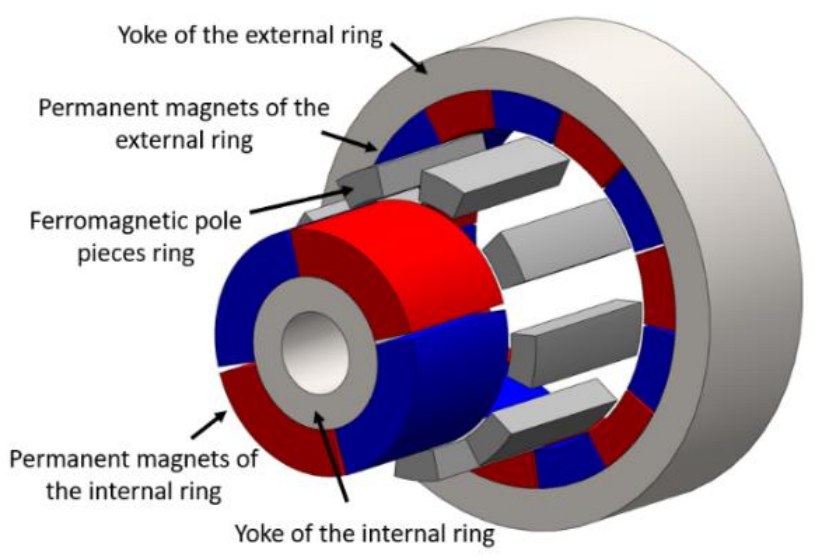

Fig. 2. Magnetic gear topology proposed by [5] in an expanded drawing with low pole numbers (in this example: $p_{\text {int }}=2, p_{\text {ext }}=7$, and $Q=9$ )

The magnetic gear topology [5] is composed of: an internal ring with $p_{\text {int }}$ pole pairs of permanent magnets and a ferromagnetic yoke, an external ring with $p_{\text {ext }}$ pole pairs of permanent magnets and a ferromagnetic yoke, and a ring with $\mathrm{Q}$ ferromagnetic poles between both permanent magnet rings (an example is provided in Fig. 2 with low pole numbers so as to improve legibility: $p_{\text {int }}=2, p_{\text {ext }}=7$, and $Q=9$ ). Each permanent magnet ring generates a magnetomotive force wave in the air gaps. The ring with ferromagnetic pole pieces is intended to modulate the magnetic field in both air gaps in order to obtain common harmonics. The result is a magnetic torque with a nonzero average and power transmission.

To achieve this power transmission, the pole numbers of all three rings must satisfy Eq. (1). Depending on the fixed ring, the gear ratio $G_{m}$ is given by (2), where $\Omega_{\text {int } / 0}, \Omega_{\text {ext } / 0}$ and $\Omega_{Q / 0}$ are the speed rotations of the internal ring, external ring and pole piece ring, respectively. To compute the magnetic field distribution in the magnetic gear, the fixed ring is not needed; the analytical magneto-static model presented in the next part is thus applicable regardless of the fixed ring.

$$
\begin{gathered}
p_{\text {int }}+p_{\text {ext }}=Q \\
\left\{\begin{array}{l}
\omega_{\text {int } / 0}=0 \rightarrow G_{m}=\frac{\Omega_{\text {ext } / 0}}{\Omega_{Q / 0}}=\frac{Q}{p_{\text {ext }}} \\
\omega_{Q / 0}=0 \rightarrow G_{m}=\frac{\Omega_{\text {int } / 0}}{\Omega_{\text {ext } / 0}}=-\frac{p_{\text {ext }}}{p_{\text {int }}} \\
\omega_{\text {ext } / 0}=0 \rightarrow G_{m}=\frac{\Omega_{\text {int } / 0}}{\Omega_{Q / 0}}=\frac{Q}{p_{\text {int }}}
\end{array}\right.
\end{gathered}
$$

\section{Analytical Magneto-Static Model}

\section{A. Magnetic field distibution resolution in regions other than pole pieces}

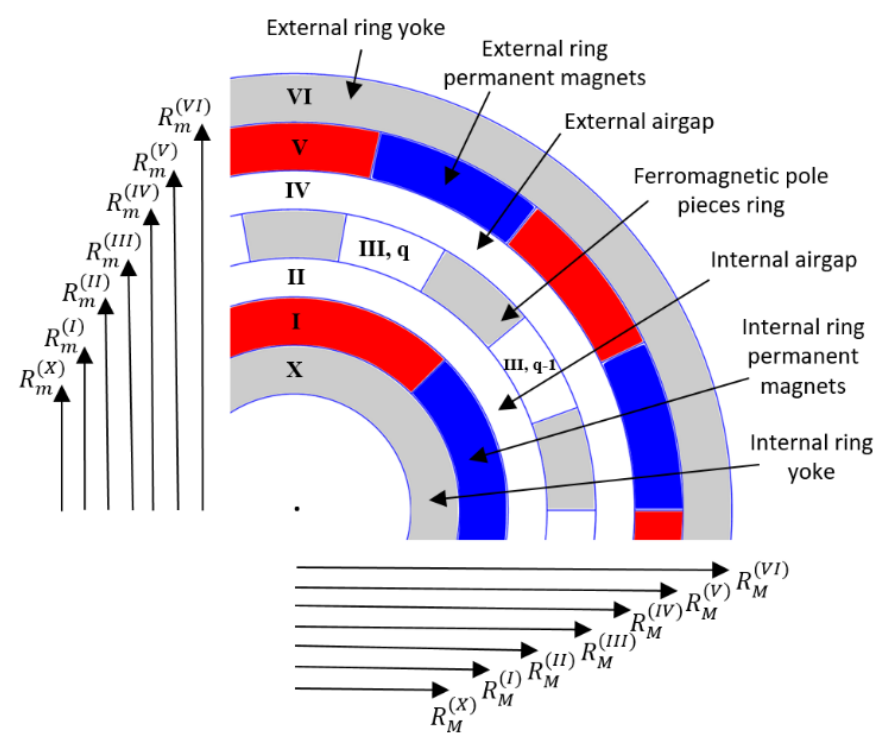

Fig. 3. Magnetic gear parameterization of the various regions (in this example: $p_{\text {int }}=2, p_{\text {ext }}=7$, and $Q=9$ )

To evaluate the iron losses in pole pieces and yokes of the magnetic gear during post-processing, it is first necessary to determine the magnetic field distribution across the various regions of the system, as shown in Fig. 3. For this computation, a 2D magneto-static model, proposed by [13] and [14], has been developed with the radial magnetization of magnets, a constant remanence of magnets and a constant relative permeability for all materials. This analytical model requires solving Poisson's 
and Laplace's equations in the $\mathrm{k}$ region of the system (3) [17] (yoke regions, permanent magnet regions, air gap regions, and each air space between pole pieces, see Fig. 3).

$$
\Delta A^{(k)}=\left\{\begin{array}{c}
-\frac{1}{r} \frac{\partial M^{(k)}}{\partial \alpha} \\
0
\end{array}\right.
$$

For Poisson's and Laplace's equations, a general solution (4) can be found in the $k$ region of the system by employing the variable separation method [18], where: $A^{(k)}$ is the magnetic vector potential, $X_{0}^{(k)}, Y_{0}^{(k)}, C_{n}^{(k)}, D_{n}^{(k)}, K_{n}^{(k)}$ and $E_{n}^{(k)}$ are integration constants, $a_{n}^{(k)}$ and $b_{n}^{(k)}$ are the general solution to Poisson's equation, $r$ and $\alpha$ are cylindrical coordinates, and $R_{M}^{(k)}=R_{m}^{(k+1)}$ are in agreement with Fig. 3. For the various problem boundaries, the corresponding conditions should be given by the equations presented in (5).

$$
\begin{aligned}
& A^{(k)}\left(r, \alpha, \theta^{(k)}\right)=X_{0}^{(k)}+Y_{0}^{(k)} \ln \left(\frac{r}{R_{M}^{(k)}}\right) \\
& +\sum_{n \geq 1}^{N}\left(C_{n}^{(k)}\left(\frac{r}{R_{M}^{(k)}}\right)^{n}+D_{n}^{(k)}\left(\frac{r}{R_{m}^{(k)}}\right)^{-n}+a_{n}^{(k)}\right) \cos (n . \alpha)(4) \\
& +\sum_{n \geq 1}^{N}\left(K_{n}^{(k)}\left(\frac{r}{R_{M}^{(k)}}\right)^{n}+E_{n}^{(k)}\left(\frac{r}{R_{m}^{(k)}}\right)^{-n}+b_{n}^{(k)}\right) \sin (n . \alpha) \\
& \left\{\begin{array}{l}
\frac{\partial A^{(k)}}{\partial r}=0 \leftarrow r=R_{M}^{(I I)}, R_{m}^{(I V)} ; \forall \alpha \in\left[\alpha_{q}+\beta, \alpha_{q+1}\right] \\
\frac{\partial A^{(I I I, q)}}{\partial \alpha}=0 \leftarrow \alpha=\alpha_{q}, \alpha_{q}+\beta ; \forall r \in\left[R_{m}^{(I I I)}, R_{M}^{(I I I)}\right] \\
\left.\frac{\mu_{(k) \cdot} \partial A^{(k)}}{\mu_{(k+1)} \cdot \partial r}\right|_{r=R_{M}^{(k)}}=\left.\frac{\partial A^{(k+1)}}{\partial r}\right|_{r=R_{m}^{(k+1)}} \leftarrow k=X \ldots V \\
\left.A^{(k)}\right|_{r=R_{m}^{(k)}}=\left.A^{(k-1)}\right|_{r=R_{M}^{(k-1)}} \leftarrow k=I \ldots V I
\end{array}\right.
\end{aligned}
$$

From these conditions, it is possible to obtain a matrix system of equations $Z$, whose integration constants indicated in (4) are the problem unknowns. The dimension of matrix $Z$, expressed in (6), is dependent on: $N_{Q}$, i.e. the number of harmonics taken into account in the air space regions between pole pieces; $Q$, the number of pole pieces; and $N$, the number of harmonics taken into account in the other regions. This matrix must be inverted to determine first the integration constants in several subdomains and then the magnetic field distribution displayed in Fig. 4. The radial flux $B_{r}^{(k)}$ and tangential flux $B_{\alpha}^{(k)}$ can be computed using (7). Comparisons may be drawn between the radial and tangential components of the flux density distribution in the middle of both the internal air gap (Fig. 5) and external air gap (Fig. 6), as obtained with the analytical model and finite element model.

$$
\operatorname{Dim}(Z)=\left(20 N+\left(2 N_{Q}+2\right) Q\right)^{2}
$$

$$
\left\{\begin{array}{l}
B_{r}^{(k)}(r, \alpha, t)=\frac{1}{r} \frac{\partial A^{(k)}}{\partial \alpha} \\
B_{\alpha}^{(k)}(r, \alpha, t)=-\frac{\partial A^{(k)}}{\partial r}
\end{array}\right.
$$

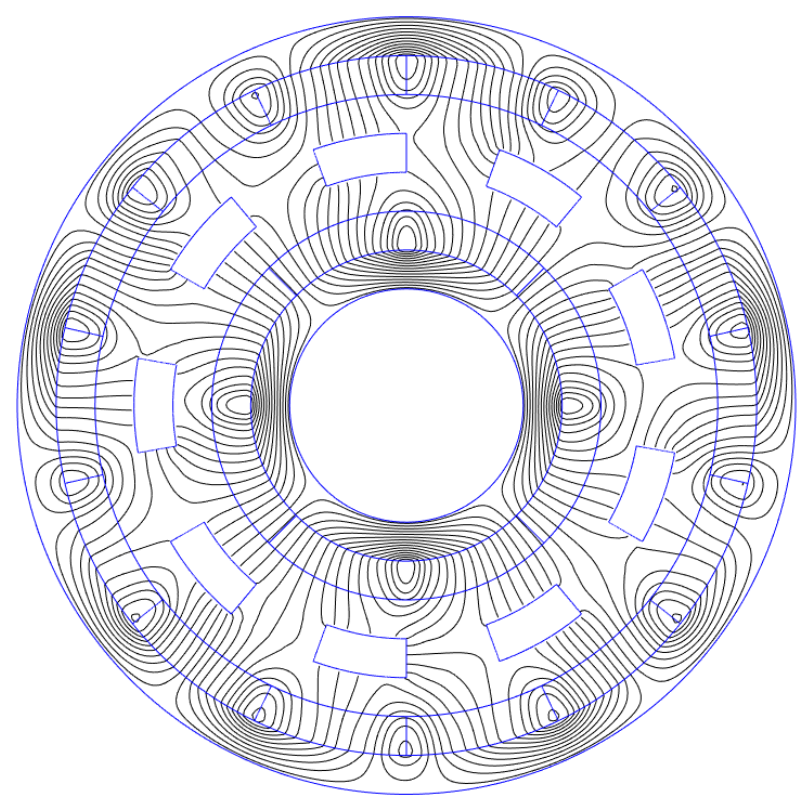

Fig. 4. Magnetic flux line distribution in a magnetic gear, as obtained with the analytical model in [14]. In this example: $p_{\text {int }}=2, p_{\text {ext }}=7$, and $Q=9$.

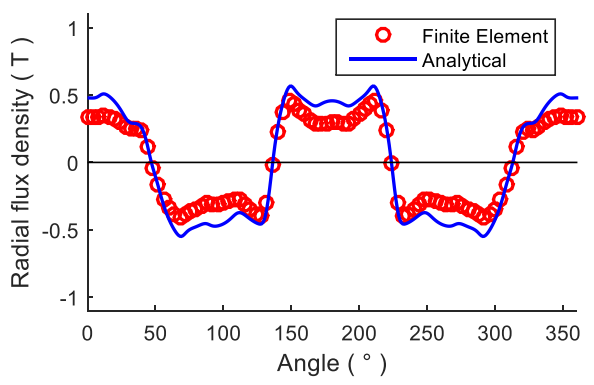

(a)

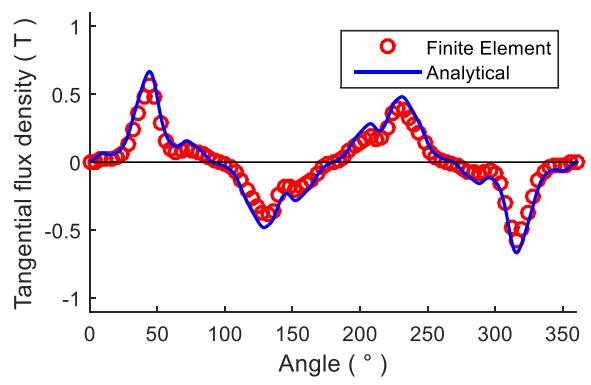

(b)

Fig. 5. Flux density distribution in the middle of the internal air gap: a) radial component, and b) tangential component 


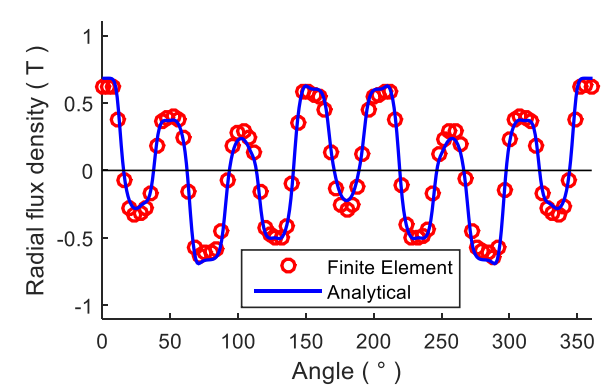

(a)

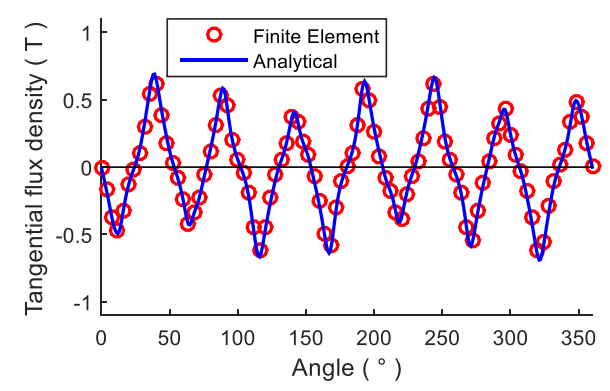

(b)

Fig. 6. Flux density distribution in the middle of the external air gap: a) radial component, and b) tangential component

With this analytical model, the magnetic field distribution is computed for just a single global position of the magnetic gear. To compute iron losses like in [16], it then becomes necessary to compute many times over the magnetic field distribution for various global positions, representing one magnetic cycle of the system. As regards iron losses in the pole pieces, since the analytical model precludes any magnetic field computation in these regions, coupling with a bi-directional reluctance network model is required to determine the flux density distribution.

\section{B. Magnetic field distribution in a pole piece resulting from coupling with a permeance network model}

To determine iron losses in pole pieces like in [16], it is possible for only one ferromagnetic pole piece to determine the flux density distribution resulting from coupling between the analytical model [14] and a bi-directional permeance network model [15], as shown in Fig. 7 with a low permeance number. A constant radial and orthoradial mesh has been adopted from (8), and the radial and tangential permeance values are computed from (9).

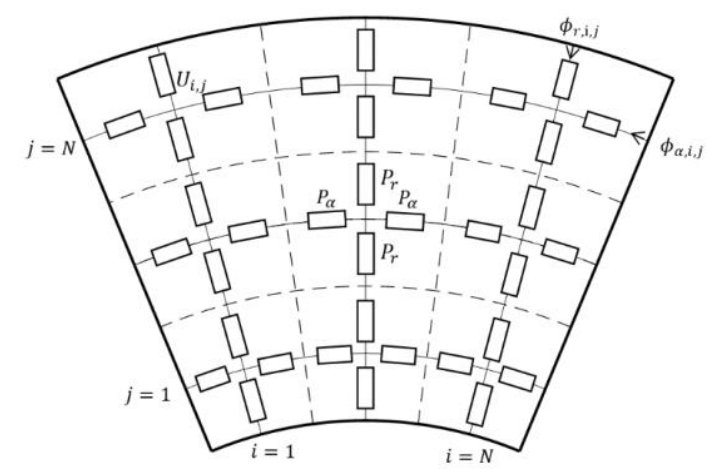

Fig. 7. Pole piece parameterization for the permeance network model

$$
\begin{gathered}
\left\{\begin{array}{c}
r_{j}=R_{m}^{(I I I)}+(j-0.5) \frac{R_{M}^{(I I I)}-R_{m}^{(I I I)}}{N} \\
\alpha_{i}=\alpha_{q}-(i-0.5) \frac{\beta}{N} \\
P_{r}=\mu_{(I I I)} \frac{\frac{1}{2} \cdot \frac{R_{m}^{(I I I)} \frac{\beta}{N}-R_{m}^{(I I I)}}{N} L}{P_{\alpha}=\mu_{(I I I)} \frac{\frac{R_{m a x}^{(I I I)}-R_{\min }^{(I I I)}}{N}}{\frac{1}{2} \cdot R_{m o y}^{(I I I)} \frac{\beta}{N}} L}
\end{array}\right.
\end{gathered}
$$

The flux sources $\phi_{i, j}$ of the bi-directional reluctance network model are determined on the pole piece boundaries from the analytical model presented above with (10), where $B_{r}^{(k)}$ and $B_{\alpha}^{(k)}$ correspond to the magnetic field determined in regions II, III and IV.

$$
\left\{\begin{array}{l}
\phi_{r, i, j}=\int B_{r}^{(k)}\left(r_{j}, \alpha_{i}\right) \cdot d S_{i, j} \\
\phi_{\alpha, i, j}=\int B_{\alpha}^{(k)}\left(r_{j}, \alpha_{i}\right) \cdot d S_{i, j}
\end{array}\right.
$$

To determine the magnetic field for the various permeance network components, it is necessary to solve equation (11) on the different nodes (the potentials $U_{i, j}$ are the unknowns of this problem). If a node is adjacent to the pole piece boundaries, a number of terms in (11) must be substituted by the flux sources determined by the previous analytical model, like in (12) if $i=$ 1 or $i=N$ and in (13) if $j=1$ or $j=N$.

$$
\begin{gathered}
\left(U_{i, j}-U_{i-1, j}\right) 2 \cdot P_{\alpha}+\left(U_{i, j}-U_{i, j-1}\right) 2 \cdot P_{r}+ \\
\left(U_{i, j}-U_{i+1, j}\right) 2 \cdot P_{\alpha}+\left(U_{i, j}-U_{i, j+1}\right) 2 \cdot P_{r}=0 \\
\left\{\begin{array}{l}
\left(U_{i, j}-U_{i-1, j}\right) 2 \cdot P_{\alpha}=\phi_{\alpha, i, j} \leftarrow i=1 \\
\left(U_{i, j}-U_{i+1, j}\right) 2 \cdot P_{\alpha}=\phi_{\alpha, i, j} \leftarrow i=N
\end{array}\right. \\
\left\{\begin{array}{l}
\left(U_{i, j}-U_{i, j-1}\right) 2 \cdot P_{r}=\phi_{r, i, j} \leftarrow j=1 \\
\left(U_{i, j}-U_{i, j+1}\right) 2 \cdot P_{r}=\phi_{r, i, j} \leftarrow j=N
\end{array}\right.
\end{gathered}
$$

From these equations, it is possible to derive a matrix system of equations (14), where: $[U]$ is the potential matrix (containing the problem unknowns), $[P]$ the permeance matrix, and $[\phi]$ the flux matrix (containing the flux determined from the analytical model). If the permeance matrix $[P]$ is inverted, then: the potential matrix $[U]$ can be determined; the radial flux $B_{r, i, j}$ and tangential flux $B_{\alpha, i, j}$ can be computed with (15); and a flux density distribution can be computed for a pole piece (see Fig. 8).

$$
[P]_{N * N, N * N} *[U]_{N * N, 1}=[\phi]_{N * N, 1}
$$




$$
\left\{\begin{array}{c}
B_{r, i, j}=\frac{1}{r_{i} \frac{\beta}{N} L}\left(\begin{array}{c}
\left(U_{i, j}-U_{i, j-1}\right) 2 . P_{r}+ \\
\left(U_{i, j+1}-U_{i, j}\right) 2 . P_{r}
\end{array}\right) \\
B_{\alpha, i, j}=\frac{1}{\frac{R_{M}^{(I I I)}-R_{m}^{(I I I)}}{N} L}\left(\begin{array}{c}
\left(U_{i, j}-U_{i-1, j}\right) 2 . P_{\alpha}+ \\
\left(U_{i+1, j}-U_{i, j}\right) 2 . P_{\alpha}
\end{array}\right)
\end{array}\right.
$$

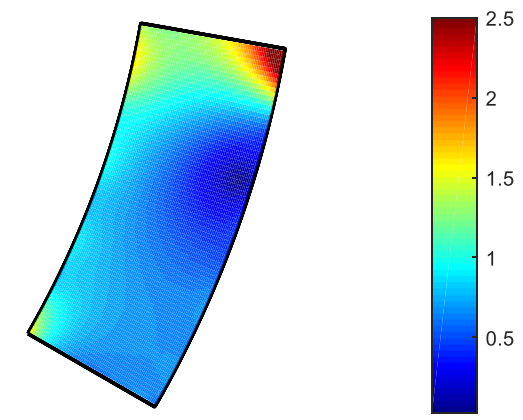

Fig. 8. Flux density in a pole piece obtained from the permeance network model

\section{FluX Density EVOLUtion In FERROMAGNETIC PARTS}

Iron losses are generated by the temporal and spatial variations in flux density across the distinct ferromagnetic regions. These flux density variations create hysteresis and eddy currents in materials, which in turn cause losses and then warm the materials. A good indication of whether or not a ferromagnetic region will generate losses entails drawing the flux density evolution at a point of the ferromagnetic part in a $B_{r}, B_{\alpha} 2 \mathrm{D}$ plane. This representation leads to observing a locus with respect to the flux density evolution. For a specific point of a ferromagnetic part, iron losses will increase with the locus amplitude and frequency of occurrence. It is thus important to analyze the locus of the various ferromagnetic parts of the magnetic gear in order to better understand the iron loss evolution. The flux density evolution will be analyzed at three distinct points shown in Fig. 9, representing the magnetic field evolution of the three regions.

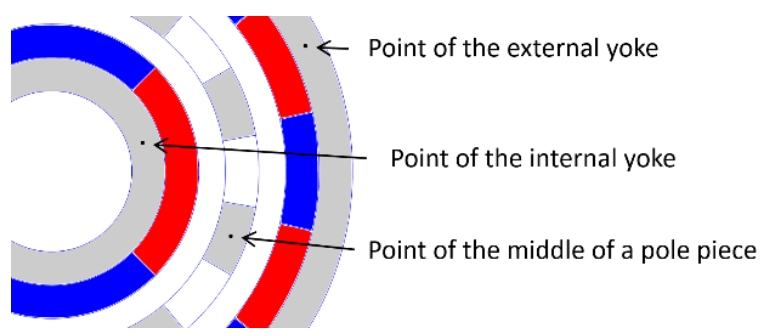

Fig. 9. Designation of the three distinct points studied in Section V, representing the magnetic field evolution of the three regions

\section{A. Locus frequency across the various ferromagnetic regions}

The frequencies of locus generation are not the same for the internal yoke, the pole pieces and the external yoke. In fact, when the magnetic gear is in rotation and when the fixed ring is the pole pieces ring, Fig. 10 shows the evolution in radial and tangential flux for the three points designated in Fig. 9 (Fig. 10a corresponds to the point of the internal yoke, $10 \mathrm{~b}$ to the point of the pole piece, and 10c to the point of the external yoke).

The frequency of locus generation for the various ferromagnetic parts thus depends on the rotational speed and pole configuration, as in Equation (16). The internal yoke locus frequency is therefore higher than the external yoke locus frequency, which in turn is higher than the pole piece locus frequency, like in (17). These frequencies must be taken into account in the iron loss computation.
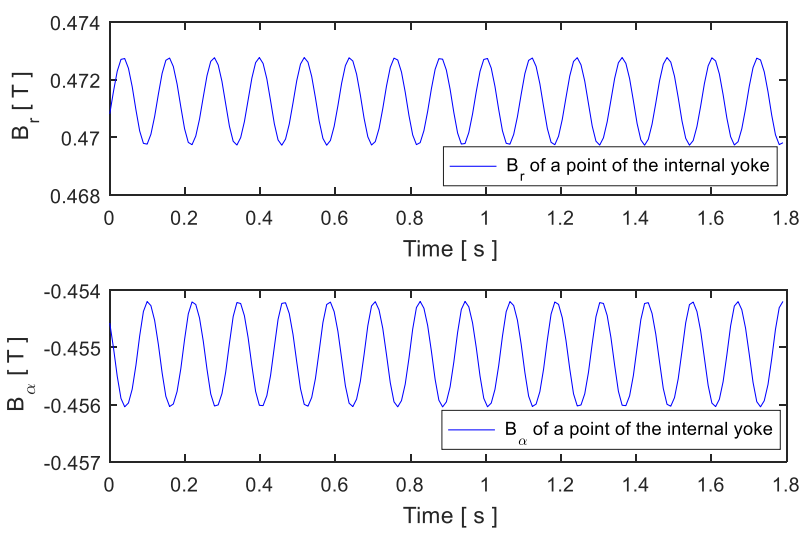

(a)
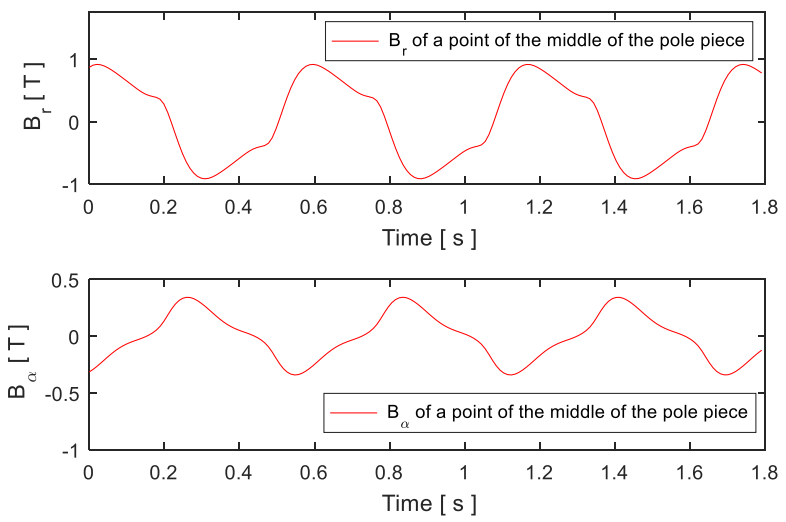

(b)
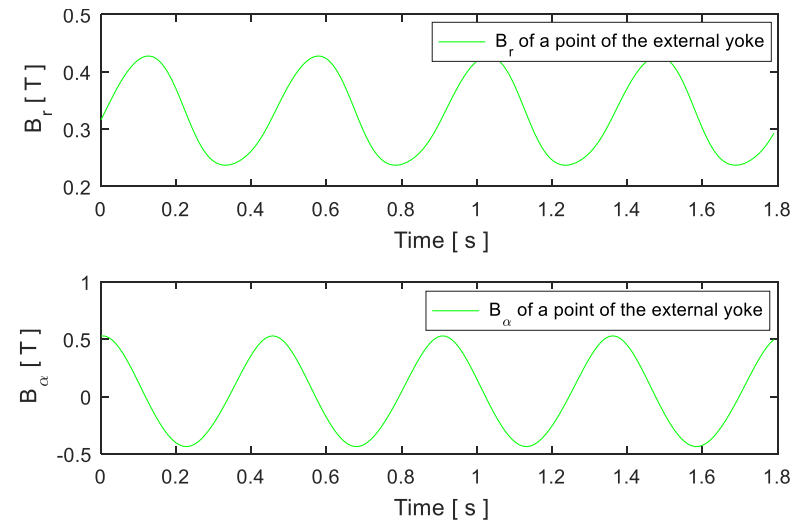

(c)

Fig. 10. Evolution in the radial and tangential components of flux density for a point on the: a) internal yoke, b) pole piece, and c) external yoke displayed in Fig. 9 


$$
\left\{\begin{array}{c}
F_{\text {int }}=\frac{p_{\text {int }} \cdot \Omega_{\text {int } / 0}}{2 \pi} \cdot \frac{Q}{p_{\text {int }}}=\frac{p_{\text {ext }} \cdot \Omega_{\text {ext } / 0}}{2 \pi} \cdot \frac{Q}{p_{\text {int }}} \\
F_{Q}=\frac{p_{\text {int }} \cdot \Omega_{\text {int } / 0}}{2 \pi}=\frac{p_{\text {ext }} \cdot \Omega_{\text {ext } / 0}}{2 \pi} \\
F_{\text {ext }}=\frac{p_{\text {int }} \cdot \Omega_{\text {int } / 0}}{2 \pi} \cdot \frac{Q}{p_{\text {ext }}}=\frac{p_{\text {ext }} \cdot \Omega_{\text {ext } / 0}}{2 \pi} \cdot \frac{Q}{p_{\text {ext }}} \\
F_{Q}<F_{\text {ext }}<F_{\text {int }}
\end{array}\right.
$$

\section{B. Locus amplitude in the various ferromagnetic regions}

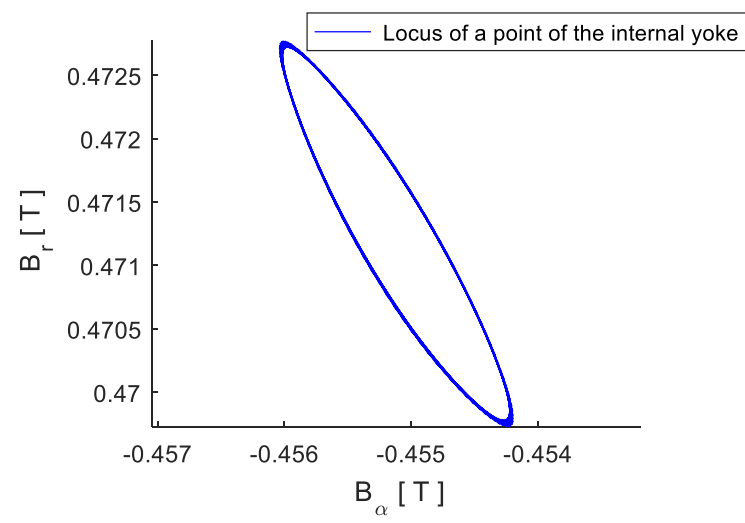

(a)

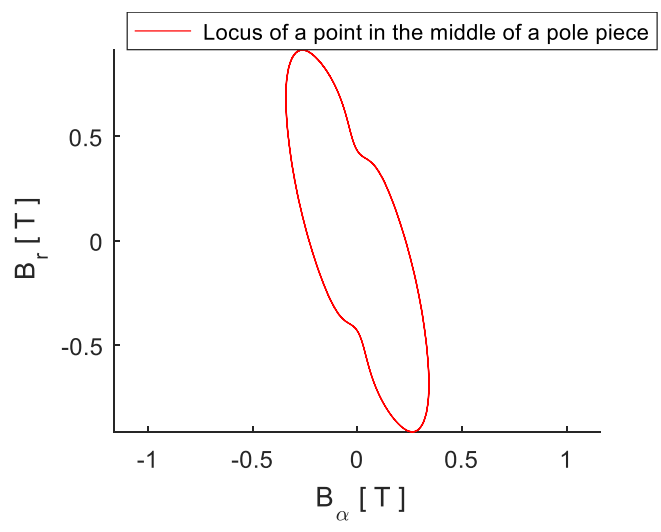

(b)

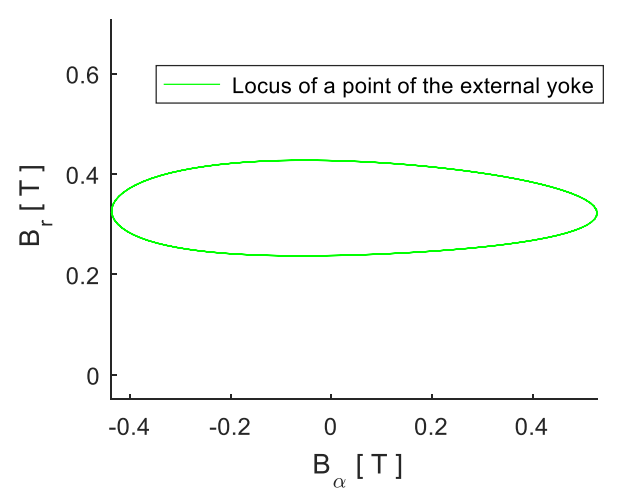

(c)
From the curve plotted in Fig. 10, the locus for the various points indicated in Fig.9 can be found, as shown in Fig.11 (Fig. 11a refers to the point in the internal yoke, $11 \mathrm{~b}$ to the point in the middle of a pole piece, and 11c to the point in the external yoke). These three loci are representative of the magnetic field evolution in these three regions. The spatial variations of flux density displayed in Fig. 11 confirm the need to include spatial variation in the iron loss computation method.

Fig. 11 shows that the locus obtained in the pole piece is higher than that found in the external yoke. This result comes from the fact that pole pieces are subjected to a rotating magnetic field, imposed by permanent magnets, as opposed to the yoke regions. This figure also reveals that the locus obtained in the external yoke is higher than that in the internal yoke due to the magnetic field distribution apparently being imposed by the ring with a low pole number of permanent magnets (i.e. the internal ring). Even though the frequency is greater for the locus with the smaller amplitude (16), iron losses in pole pieces will still be higher than losses in either the external or internal yoke.

\section{IRON LOSS COMPUTATION}

The studies in this section have been based on an example with a high pole number, as described in Table 2, which corresponds to a magnetic gear optimized for wind applications similar to that in [4] with the iron loss coefficient proposed in [19] for a laminated steel grade M1000-65.

TABle I: Data of the Magnetic Gear EXAMPLE IN SeCtion VI

\begin{tabular}{|c|c|c|}
\hline Symbol & Quantity & Value \\
\hline$P_{\text {rated }}$ & Rated power transmitted & $3.9 \mathrm{MW}$ \\
\hline$N_{l}$ & Rated speed of the low-speed rotor & $15 \mathrm{rpm}$ \\
\hline$N_{h}$ & Rated speed of the high-speed rotor & $97 \mathrm{rpm}$ \\
\hline$p_{\text {int }}$ & Number of internal ring pole pairs & 20 \\
\hline$p_{e x t}$ & Number of external ring pole pairs & 131 \\
\hline$Q$ & Number of ferromagnetic pole pieces & 151 \\
\hline$D$ & External diameter & $4 \mathrm{~m}$ \\
\hline$L_{z}$ & Magnetic length & $2 \mathrm{~m}$ \\
\hline$\delta_{\text {int }}$ & Internal air gap & $5 \mathrm{~mm}$ \\
\hline$\delta_{e x t}$ & External air gap & $5 \mathrm{~mm}$ \\
\hline$B_{r e m}$ & Remanence of the magnets & $1.20 \mathrm{~T}$ \\
\hline$\mu_{(I, V)}$ & Relative permeability of magnets & 1 \\
\hline$\mu_{(X, I I I, V I)}$ & Relative permeability of iron & 1,000 \\
\hline$k_{h}$ & Hysteresis coefficient & 363.78 \\
\hline$k_{e}$ & Eddy current coefficient & 0.167 \\
\hline$k_{e x}$ & Excess loss coefficient & 1.84 \\
\hline$\gamma$ & Steinmetz coefficient & 2 \\
\hline
\end{tabular}

Fig. 11. Spatial variations in flux density at a point of the: a) internal yoke, b) pole piece, and c) external yoke (presented in Fig. 9) 


\section{A. Analytical iron loss model}

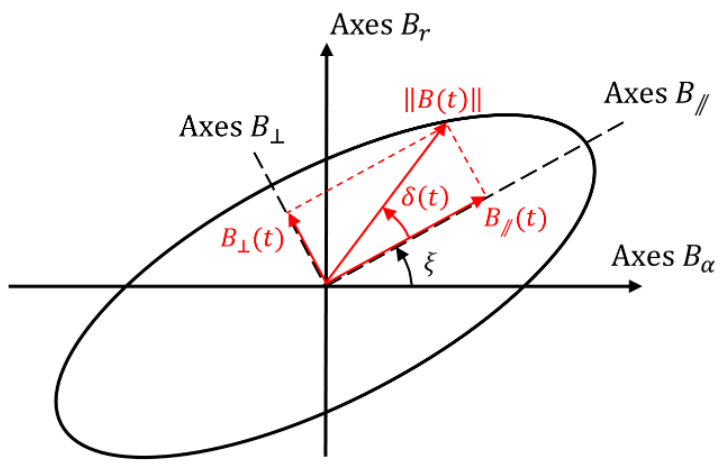

Fig. 12. Locus parameterization for the iron loss evaluation

To evaluate the iron losses in yokes and pole pieces of the magnetic gear, both $B_{/ /}$the major axis of the flux density locus and $B_{\perp}$ the minor axis of the flux density locus, as defined Fig. 12 , must be determined [16]. It is thus necessary to compute the magnetic field distribution using the global analytical model presented above (Poisson's and Laplace's resolution model coupled with the permeance network model) for various positions of the magnetic gear, i.e. representative of a magnetic cycle. The next step first consists of evaluating the norm of the flux (18) everywhere in the ferromagnetic parts:

$$
\begin{aligned}
& \left\|B\left(r_{j}, \alpha_{i}, \theta\right)\right\| \\
& =\sqrt{\begin{array}{c}
\left(B_{r}\left(r_{j}, \alpha_{i}+\theta\right)-B_{r \text { moy }}\left(r_{j}, \alpha_{i}\right)\right)^{2} \\
+\left(B_{\alpha}\left(r_{j}, \alpha_{i}+\theta\right)-B_{\alpha \text { moy }}\left(r_{j}, \alpha_{i}\right)\right)^{2}
\end{array}}
\end{aligned}
$$

For the various points $\left(r_{j}, \alpha_{i}\right)$ of the ferromagnetic parts, it then becomes possible to evaluate the position of the magnetic gear $\theta_{i, j}^{*}$ that maximizes the norm of the flux density. The major axis angle of the flux density $\xi_{i, j}$ (as defined in Fig. 12) must be determined from (19) for points $\left(r_{j}, \alpha_{i}\right)$ [20]. From this angle, $B_{/ /}$and $B_{\perp}$ are determined from Eq. (20), where $\delta(r, \alpha, t)$ is defined in Fig. 12 and Eq. (21).

$$
\begin{gathered}
\xi_{i, j}=\arctan \left(\frac{B_{r}\left(\theta_{i, j}^{*}\right)}{B_{\alpha}\left(\theta_{i, j}^{*}\right)}\right) \\
\left\{\begin{array}{l}
B_{/ /}\left(r_{j}, \alpha_{i}, \theta\right)=\left\|B\left(r_{j}, \alpha_{i}, \theta\right)\right\| \cdot \cos \left(\delta_{i, j}(\theta)\right) \\
B_{\perp}\left(r_{j}, \alpha_{i}, \theta\right)=\left\|B\left(r_{j}, \alpha_{i}, \theta\right)\right\| \cdot \sin \left(\delta_{i, j}(\theta)\right)
\end{array}\right. \\
\delta_{i, j}(\theta)=\arctan \left(\frac{B_{r}\left(r_{j}, \alpha_{i}+\theta\right)}{B_{\alpha}\left(r_{j}, \alpha_{i}+\theta\right)}\right)-\xi_{i, j}
\end{gathered}
$$

The instantaneous iron loss density $P_{\text {iron }}$ is then determined from Eq. (22), where $k_{h}, k_{e}$ and $k_{e x}$ are the hysteresis coefficient, eddy current coefficient and excess loss coefficient, respectively, and $\gamma$ the Steinmetz coefficient [21]. In Eq. (18), $F$ corresponds to: $F_{i n t}$ for the internal yoke iron loss computation,
$F_{Q}$ for the pole piece iron loss computation, and $F_{\text {ext }}$ for the external yoke iron loss computation (defined in (16)). Fig. 13 shows the evolution in iron losses for the various ferromagnetic parts as a function of the internal ring speed when the pole piece ring is stationary, for the magnetic gear described in Table I with a laminated steel grade M1000-65 (linear properties have been assumed). Values obtained at the rated power are provided in Table II. This computation is performed three times depending on the ferromagnetic region with the adapted periodicity (as defined in (16)). For these three periods, the evolution in position of the magnetic gear is dissected in 50 positions. For the three ferromagnetic regions, the flux density evolution is evaluated with both the analytical model and finite element model at multiple points (the three ferromagnetic parts are dissected at only $20 * 20$ points for a pole periodicity of this region). For this magnetic gear, the total of the iron losses correspond to $1.9 \%$ of the transmitted power.

$$
\begin{gathered}
P_{\text {iron }}\left(r_{j}, \alpha_{i}\right)=k_{h} \cdot F \cdot\left(\left(\frac{\Delta B_{\perp}}{2}\right)^{\gamma}+\left(\frac{\Delta B_{/ /}}{2}\right)^{\gamma}\right) \\
+k_{e} \frac{1}{T} \int_{t=0}^{T}\left(\left(\frac{d B_{\perp}}{d t}\right)^{2}+\left(\frac{d B_{/ /}}{d t}\right)^{2}\right) d t \\
+k_{\text {ex }} \frac{1}{T} \int_{t=0}^{T}\left(\left(\frac{d B_{\perp}}{d t}\right)^{2}+\left(\frac{d B_{/ /}}{d t}\right)^{2}\right)^{3 / 4} d t
\end{gathered}
$$

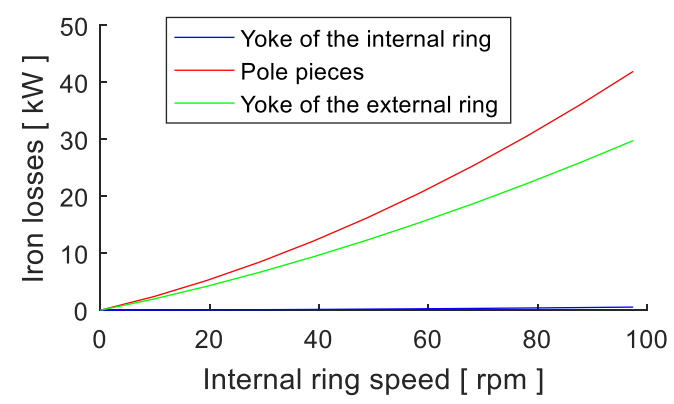

Fig. 13. Iron loss evolution in the various ferromagnetic parts as a function of internal ring speed when the pole piece ring is stationary, for the magnetic gear described in Table I

TABLE II: IRON LoSSES OBTAINNEd From the ANALYTICAL ModEL

\begin{tabular}{|c|c|}
\hline & $\begin{array}{c}\text { Losses obtained from the } \\
\text { analytical model }(\mathbf{k W})\end{array}$ \\
\hline $\begin{array}{c}\text { Internal } \\
\text { yoke }\end{array}$ & 0.62 \\
\hline $\begin{array}{c}\text { Pole } \\
\text { pieces }\end{array}$ & 44.1 \\
\hline $\begin{array}{c}\text { External } \\
\text { yoke }\end{array}$ & 31.5 \\
\hline
\end{tabular}

\section{B. Computation time benefit}

The post-processing computation time is the same for the iron loss analytical model and the finite element model. It is a negligible part of the global computation time. For both models, the major part of the global computation time corresponds to the magnetic field repartition resolution for every dissected position 
of the magnetic gear. Considering the harmonic selection method proposed in [14] for the Laplace's and Poisson's equations and the permeance network presented above (with a pole piece dissected in $20 * 20$ points), the magnetic field computation time for only one position is 1.5 seconds for the analytical model vs. 300 seconds with the finite element model (for the magnetic gear presented in Table I).

Then, when the position evolution of the magnetic gear is dissected in 50 positions, the global computation time necessary to evaluate iron loss in the internal and external yokes and in the pole pieces is 90 seconds with the analytical model vs. 4 hours with the finite element model. The analytical model thus permits to divide the computation time by 150 .

\section{CONCLUSION}

This article has focused on a fast analytical model of iron losses in the ferromagnetic parts of the magnetic gear (i.e. internal yoke, external yoke and pole pieces). The proposed $2 \mathrm{D}$ magneto-static analytical linear model has been based on a resolution of both Laplace's and Poisson's equations coupled with a permeance network, in order to determine the magnetic field distribution in pole pieces. The iron loss model introduced in this article [16] takes into account both the temporal and spatial variations of flux density.

For the high-power magnetic gear described in [4] and describe in table I, the iron loss has been computed in 90 seconds with the analytical model proposed in this article vs. 4 hours if the magnetic field is resolved using the finite element model.

Looking forward, results from this iron loss model must be compared to those obtained from a magnetic field resolution with a finite element model. A comparison between results obtained from the iron loss computation method in considering spatial variation and those found without considering spatial variation can also be drawn. As a next step, a computation time analysis can be conducted in order to reduce the time required to compute the losses by varying both the number of points taken into consideration in the three regions and the number of magnetic gear positions. In the near future, this fast iron loss computation model will be integrated into a global mechatronic optimization of the magnetic gear. Such an optimization process will also include an analytical model of eddy current losses in permanent magnets [22].

\section{ACKNOWLEDGMENTS}

The authors would like to thank Jeumont Electric for the various discussions held on system design, with special gratitude to D. Laloy, D. Ekeom and A. Fasquelle.

\section{REFERENCES}

[1] R. Lacal Arántegui and J. Serrano Gonzáles, 2014 JRC wind status report: Technology, market and economic aspects of wind energy in Europe. 2015.

[2] J. Keller, Y. Guo, and L. Sethuraman, "Gearbox Reliability Collaborative Investigation of Gearbox Motion and High-SpeedShaft Loads," NREL, Tech. Rep. TP-5000-65321, 2016.
[3] W. Teng, X. Ding, X. Zhang, Y. Liu, and Z. Ma, "Multi-fault detection and failure analysis of wind turbine gearbox using complex wavelet transform," Renew. Energy, vol. 93, pp. 591-598, 2016.

[4] M. Desvaux, R. L. G. Latimier, B. Multon, H. Ben Ahmed, and S. Sire, "Design and optimization of magnetic gears with arrangement and mechanical constraints for wind turbine applications," in 2016 11th International Conference on Ecological Vehicles and Renewable Energies, EVER 2016, 2016.

[5] T. B. Martin, "Magnetic transmission," Pat. US3378710, 1968.

[6] K. Atallah and D. Howe, "A novel high-performance magnetic gear," IEEE Trans. Magn., vol. 37, no. 4, pp. 2844-2846, 2001.

[7] K. Atallah, S. D. Calverley, and D. Howe, "Design, analysis and realisation of a high-performance magnetic gear," IEE Proc - Electr. Power Appl., vol. 151, no. 2, pp. 135-143, 2004.

[8] E. Gouda, S. Mezani, L. Baghli, and A. Rezzoug, "Comparative study between mechanical and magnetic planetary gears," IEEE Trans. Magn., vol. 47, no. 2, pp. 439-450, 2011.

[9] A. Penzkofer and K. Atallah, "Magnetic Gears for High Torque Applications," IEEE Trans. Magn., vol. 50, no. 11, 2014.

[10] A. S. Bornschlegell, J. Pelle, S. Harmand, A. Fasquelle, and J. P. Corriou, "Thermal optimization of a high-power salient-pole electrical machine," IEEE Trans. Ind. Electron., vol. 60, no. 5, pp. 1734-1746, 2013.

[11] P. O. Rasmussen, T. V. Frandsen, K. K. Jensen, and K. Jessen, "Experimental Evaluation of a Motor Integrated Permanent Magnet Gear," in IEEE Energy Conversion Congress and Exposition (ECCE 2011), 2011, pp. 3982-3989.

[12] L. Jian, S. Member, K. T. Chau, and S. Member, "A Coaxial Magnetic Gear With Halbach Permanent-Magnet Arrays," IEEE Trans. Energy Convers., vol. 25, no. 2, pp. 319-328, 2010.

[13] T. Lubin, S. Mezani, and A. Rezzoug, "Analytical computation of the magnetic field distribution in a magnetic gear," IEEE Trans. Magn., vol. 46, no. 7, pp. 2611-2621, 2010.

[14] M. Desvaux, B. Traullé, R. L. G. Latimier, S. Sire, B. Multon, and H. Ben Ahmed, "Computation Time Analysis of the Magnetic Gear Analytical Model," IEEE Trans. Magn. , Accept., 2017.

[15] V. Ostović, "Dynamics of Saturated Electric Machines," in New York Springer-Verlag, 1989.

[16] C. A. Hernandez-Aramburo, T. C. Green, and A. C. Smith, "Estimating Rotational Iron Losses in an Induction Machine," IEEE Trans. Magn., vol. 39, no. 6, pp. 3527-3533, 2003.

[17] J. G. Van Bladel, "Electromagnetic Fields: Second Edition," Electromagn. Fields Second Ed., pp. 1-1147, 2006.

[18] S. J. Farlow, Partial Differential Equations for Scientists and Engineers, Dover Publ. 1982.

[19] M. Fratila, A. Benabou, A. Tounzi, and M. Dessoude, "Calculation of Iron Losses in Solid Rotor Induction Machine Using FEM," IEEE Trans. Magn., vol. 50, no. 2, pp. 2-5, 2014.

[20] G. Bertotti, a. Boglietti, M. Chiampi, D. Chiarabaglio, F. Fiorillo, and M. Lazzari, "An improved estimation of iron losses in rotatinf electrical machines," IEEE Trans. Magn., vol. 27, no. 6, pp. 50075009, 1991.

[21] F. Deng, "An improved iron loss estimation for permanent magnet brushless machines," IEEE Trans. Energy Convers., vol. 14, no. 4, pp. 1391-1395, 1999.

[22] M. Desvaux, R. Le Goff Latimier, H. Ben Ahmed, B. Multon, and S. Sire, "Modélisation des pertes dans les aimants des multiplicateurs magnétiques pour l'éolien," in Symposium de Génie Electrique (SGE 2016), 2016 